\title{
Enhancement of capecitabine efficacy by oxaliplatin in human colorectal and gastric cancer xenografts
}

\author{
NORIAKI SAWADA, KUMIKO KONDOH and KAZUSHIGE MORI \\ Product Research Department, Kamakura Research Center, Chugai Pharmaceutical Co., Ltd., \\ 200 Kajiwara, Kamakura, Kanagawa 247-8530, Japan
}

Received May 11, 2007; Accepted July 9, 2007

\begin{abstract}
We have evaluated the antitumor activity of XELOX (a combination therapy of capecitabine $\left(\mathrm{Xeloda}^{\circledR}\right)$ and oxaliplatin) in human colorectal and gastric cancer xenograft models. In human colorectal cancer model CXF280, antitumor activity of the combination at two-thirds of the maximum tolerated dose (MTD) was superior to that of each monotherapy at MTD. Furthermore, in human colorectal cancer model COL-05-JCK and human gastric cancer xenograft model GXF 97, the combination also showed at least additive antitumor activity. In addition, toxicity was not augmented with the combination therapy in these three models. As demonstrated using ELISA or immunohistochemistry, oxaliplatin in xenograft model tumors up-regulated the level of thymidine phosphorylase (dThdPase), a key enzyme for the metabolism of capecitabine to 5-fluorouracil. These results suggest that oxaliplatin might potentiate the antitumor activity of capecitabine by up-regulating the tumor level of dThdPase. Based on these results, clinical trials of XELOX against colorectal and gastric cancers are warranted.
\end{abstract}

\section{Introduction}

Capecitabine $\left(\right.$ Xeloda $\left.^{\circledR}\right)$ is one of the oral 5-fluropyrimidine (5-FUra) derivatives and generates the active drug 5-FUra in tumors by a three-step cascade of enzymes located in the liver and tumor. The final step is the conversion of 5'-deoxyfluorouridine, an intermediated metabolite of capecitabine, to 5-FUra by thymidine phosphorylase (dThdPase) in tumors (1). In clinical studies, capecitabine has been shown to be a highly active and better-tolerated alternative to 5-FUra plus leucovorin (LV) in colorectal cancers (2-4). In human cancer xenograft models, susceptibility to capecitabine has been reported to correlate with levels of dThdPase in tumor, which is not the case with 5-FUra (5). Furthermore, it has also been reported that capecitabine shows superior antitumor activity

Correspondence to: Noriaki Sawada, Product Research Department, Kamakura Research Center, Chugai Pharmaceutical Co., Ltd., 200 Kajiwara, Kamakura, Kanagawa 247-8530, Japan

E-mail: sawadanra@chugai-pharm.co.jp

Key words: capecitabine, oxaliplatin, colorectal cancer, gastric cancer, thymidine phosphorylase in combination with dThdPase up-regulators, such as taxanes and X-ray irradiation (6-8).

Oxaliplatin is a third-generation platinum drug and, in combination therapy with 5-FUra/LV, was superior to 5-FUra/ $\mathrm{LV}$ alone in first- and second-line treatments for metastatic colorectal cancer $(9,10)$. Additionally, first-line therapy with the combination of capecitabine and oxaliplatin (XELOX) has shown a good response rate and favorable progressionfree survival (PFS) in a Phase II study of metastatic colorectal cancers (11).

In the present study with gastrointestinal cancer xenograft models, the XELOX combination showed superior antitumor activity over each single agent. In addition, oxaliplatin upregulated thymidine phosphorylase (dThdPase) in tumor tissues and thus would potentiate antitumor activity of capecitabine. These results suggest that oxaliplatin in partnership with capecitabine would be an effective combination therapy for the treatment of gastrointestinal cancers.

\section{Materials and methods}

Chemicals. Capecitabine was synthesized at Hoffmann-La Roche (Basel, Switzerland). Oxaliplatin was provided by Sanofi-Synthelabo (PA, USA).

Capecitabine was dissolved or suspended in $40 \mathrm{mM}$ citrate buffer ( $\mathrm{pH}$ 6.0) containing gum arabic and then administered orally once a day for 14 days followed by a week of rest. Oxaliplatin was dissolved in 5\% glucose and administered intravenously on Day 1 . In the human gastric cancer xenograft model, capecitabine was administered from Day 1 to 14 and Day 22 to Day 35, and oxaliplatin was injected on Day 1 and Day 22.

Animals. Four- or 5-week-old BALB/c nu/nu mice obtained from Charles River (Yokohama, Japan) and observed for at least 1 week were used in the experiments. All animal experiments were conducted in accordance with the guidelines for the Care and Use of Laboratory Animals in Nippon Roche Research Center (the former name of the affiliate of the authors).

Tumors. The human tumor lines used were the colorectal cancer line CXF280 and gastric cancer line GXF97 provided by Professor H.H. Fiebig (Freiberg University, Freiberg, Germany) and the colorectal cancer line COL-05-JCK from the Central Institute for Experimental Animals (Kanagawa, 
Japan). All lines were maintained by continuous passage with pieces of tumor inoculated subcutaneously in the BALB/c nu/nu mice.

Human cancer xenograft models. A small piece of CXF280, COL-05-JCK, or GXF97 was inoculated subcutaneously into the right flank of a nude mouse. Administration of the drug started on the day when the mean volume of the tumors reached $\sim 300-450 \mathrm{~mm}^{3}$. Tumor volume $(\mathrm{V})$ was estimated using the equation $\mathrm{V}=a b^{2} / 2$, where $a$ and $b$ are tumor length and width, respectively. In the combination studies, mice bearing CXF280 or GXF97 were randomized into 6 mice per group and treated with the drugs. Mice bearing COL-05JCK were randomized into 8 mice per group. To evaluate the efficacy of the drugs, tumor size and body weight were measured twice a week. In order to determine the levels of dThdPase in tumor, and for the immunohistochemical study, mice bearing CXF280 or COL-05-JCK were randomized into 4 mice per group and treated once with oxaliplatin. The tumors were excised 4 or 7 days after the drug administration to measure the dThdPase levels.

Determination of tumor levels of dThdPase. Tumor tissues were homogenized in $10 \mathrm{mM}$ Tris-buffer ( $\mathrm{pH}$ 7.4) containing $15 \mathrm{mM} \mathrm{NaCl}, 1.5 \mathrm{mM} \mathrm{MgCl}_{2}$, and $50 \mu \mathrm{M}$ potassium phosphate using a glass homogenizer. The homogenate was then centrifuged at $10,000 \mathrm{~g}$ for $20 \mathrm{~min}$ at $4^{\circ} \mathrm{C}$, and the supernatants were stored at $-80^{\circ} \mathrm{C}$ until use. The protein concentration of the supernatants was determined using a DC protein assay kit (Bio-Rad). The level of dThdPase was measured by ELISA with monoclonal antibodies specific to human dThdPase as previously described by Nishida et al (12). One unit corresponds to the amount of dThdPase of a standard enzyme solution (extracts from human colon cancer xenograft HCT116) which phosphorolyzes 5'-DFUR to 5-FU at a rate of $1 \mu \mathrm{g}$ 5-FUra per $\mathrm{h}$. The ELISA results showed no crossreactivity to mouse dThdPase.

Immunohistochemistry for dThdPase. Tumor tissues from human colorectal cancer xenograft model COL-05-JCK were fixed in a $10 \%$ neutral formalin solution, dehydrated with graded concentrations of ethanol, and embedded in paraffin. Deparaffinized $2-\mu \mathrm{m}$ sections were washed in phosphatebuffer saline (PBS) for $5 \mathrm{~min}$ three times and steamed in a preheated steamer for $20 \mathrm{~min}$. After removal and cool down for $20 \mathrm{~min}$ at room temperature, the sections were washed twice with distilled water. The sections were then treated with $0.3 \% \mathrm{H}_{2} \mathrm{O}_{2}$ in absolute methanol to inactivate endogenous peroxidase and washed again with PBS for 5 min three times. The immunohistochemistry (IHC) method was as previously described (13). Slides were dehydrated and mounted with a non-aqueous permanent mounting medium. The anti-dThdPase IgG used was purified from rabbit antiserum by affinity column with recombinant dThdPase. As the second antibody for IHC, we used biotinylated anti-rabbit IgG antibody. The IHC-staining images of the tumor tissues were captured with a digital camera and the ratio of the area of dThdPasepositive cells to the area of tumor cells in the tumor tissues was calculated using Win Roof (Mitani Corp., Inc., Fukui, Japan).
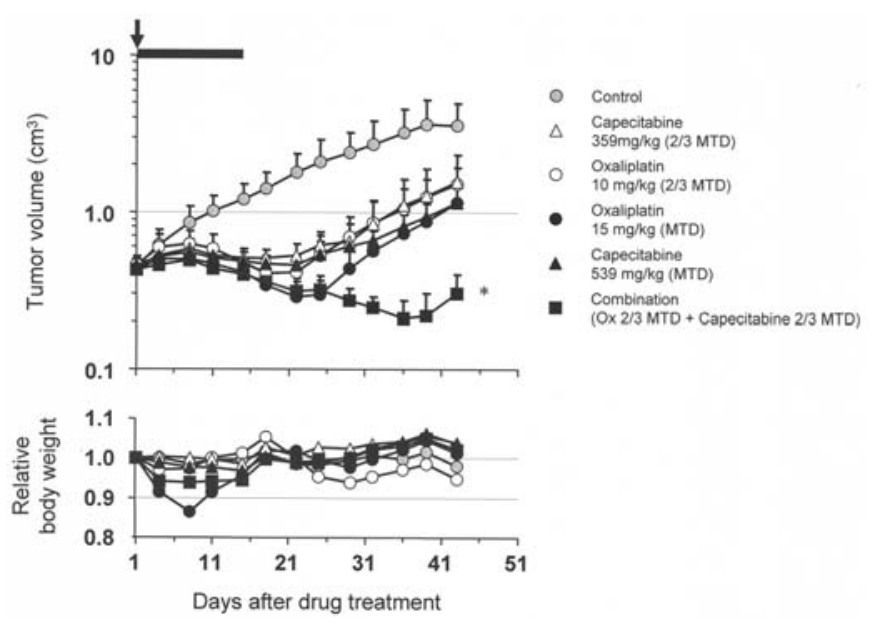

Figure 1. Antitumor activity of the combination of capecitabine and oxaliplatin in human colon cancer xenograft model CXF280. Capecitabine was administered orally once a day from Day 1 to Day 14 and oxaliplatin was injected intravenously on Day 1. Data points indicate mean values of tumor volume and relative body weight; bars indicate standard deviations. Bold horizontal bar shows the period of capecitabine administration; the arrow shows the time of oxaliplatin injection. ${ }^{*} \mathrm{p}<0.05$ vs. all single agent groups.

Statistical analysis. Tumor volume and tumor growth delay of the combination group were compared to that of the control group and the monotherapy groups using the Mann-Whitney $\mathrm{U}$ test. The level of dThdPase in tumor of the oxaliplatintreated groups and the vehicle control group were also compared using the Mann-Whitney U test. Differences were considered to be significant at $\mathrm{p}<0.05$.

\section{Results}

Combination therapy. The antitumor activity of capecitabine in combination with oxaliplatin was evaluated in human colon cancer xenograft model CXF280. The combination therapy of capecitabine at 2/3 MTD with oxaliplatin at 2/3 MTD showed significantly higher antitumor activity than each monotherapy at MTD (Fig. 1). In addition, no augmentation of toxicity, as shown by body weight loss, was observed in the combination treatment.

The combination of these drugs was also evaluated in human colon cancer xenograft model COL-05-JCK. Oxaliplatin alone showed no significant antitumor activity and no significant delay of tumor growth in this model. However, the combination therapy showed significant tumor growth delay as compared with capecitabine alone (Fig. 2), although the tumor growth inhibition estimated from tumor volume was not significant.

Furthermore, the XELOX combination showed at least additive effect in human gastric cancer xenograft model GXF97 (Fig. 3). Two of the 6 mice in the combination group had no palpable tumor one week after 2 cycles of the treatment.

dThdPase up-regulation. We measured the level of dThdPase in tumor tissues of the CXF280 and COL-05-JCK models treated with oxaliplatin using ELISA. Levels of dThdPase in tumor were significantly increased 7 days after single administration of oxaliplatin in the CXF280 model (Table I) 


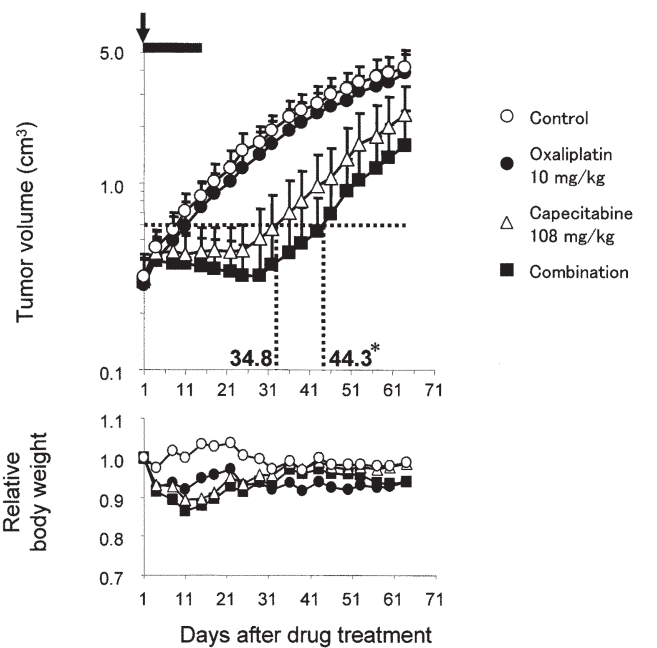

Figure 2. Antitumor activity of the combination of capecitabine and oxaliplatin in human colon cancer xenograft model COL-05-JCK. Capecitabine was administered orally once a day from Day 1 to Day 14 and oxaliplatin was injected intravenously on Day 1 . Data points indicate mean values of tumor volume and relative body weight; bars indicate standard deviations. Bold horizontal bar shows the period of capecitabine administration; the arrow shows the time of oxaliplatin injection. ${ }^{*} \mathrm{p}<0.05 \mathrm{vs}$. all single agent groups.

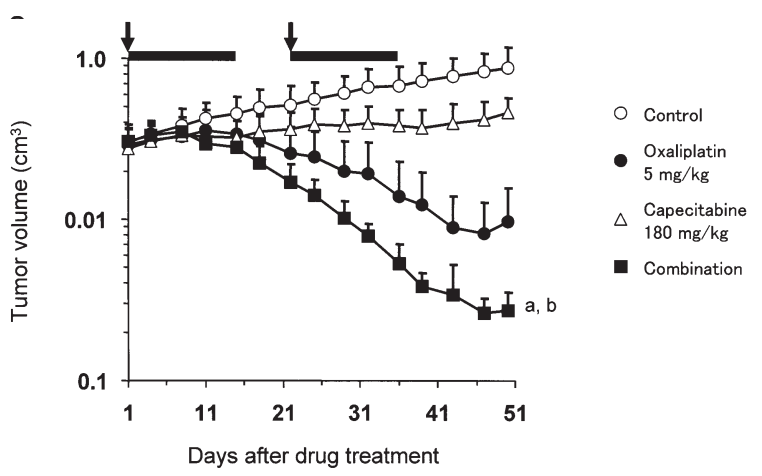

Figure 3. Antitumor activity of the combination of capecitabine and oxaliplatin in human gastric cancer xenograft model GXF97. Capecitabine was administered orally once a day from Day 1 to Day 14 and Day 22 to Day 35, and oxaliplatin was injected intravenously on Day 1 and Day 22. Data points indicate mean values of tumor volume and relative body weight; bars indicate standard deviations. Bold horizontal bars show the period of capecitabine administration; arrows show the time of oxaliplatin injection. ${ }^{\mathrm{a}} \mathrm{p}<0.05 \mathrm{vs}$. both single agent groups. ${ }^{\mathrm{b}} \mathrm{Two}$ of 6 mice had no palpable tumor.

4a) vehicle

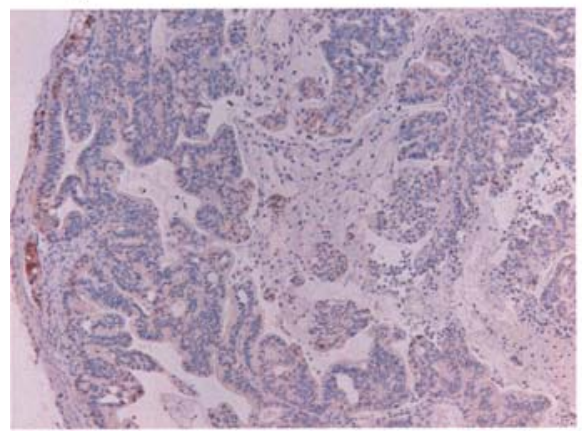

Table I. Up-regulation of dThdPase by oxaliplatin.

\begin{tabular}{lc} 
Sample & $\begin{array}{c}\text { dThdPase leve } \\
\text { (unit/mg protei }\end{array}$ \\
\hline Day 7 & \\
Vehicle & $4.3 \pm 0.2$ \\
Oxaliplatin $5 \mathrm{mg} / \mathrm{kg}$ & $9.1 \pm 1.2^{\mathrm{b}}$ \\
Oxaliplatin $10 \mathrm{mg} / \mathrm{kg}$ & $8.4 \pm 0.5^{\mathrm{b}}$ \\
Oxaliplatin $15 \mathrm{mg} / \mathrm{kg}$ & $9.4 \pm 0.5^{\mathrm{b}}$
\end{tabular}

Oxaliplatin was intravenously administered in human colon cancer xenograft model CXF280. Tumor tissues were excised to measure dThdPase levels at 7 days after administration of oxaliplatin. ${ }^{a} \mathrm{dThdPase}$ levels in tumor tissues were measured by ELISA. ' ${ }^{\mathrm{S}}$ Significantly different from the control group; $\mathrm{p}<0.05$, Mann-Whitney U-test.

On the other hand, in the COL-05-JCK model, no significant increase in dThdPase activity was observed in tumor tissue 4 or 7 days after treatment with oxaliplatin. To investigate the tumor cell expression of dThdPase in the COL-05-JCK model, we conducted an immunohistochemistry (IHC) examination. The ratio of the area of dThdPase-positive cells to the area of tumor cells in the tumor tissues after the treatment with oxaliplatin tended to be higher than that of the vehicle control (Table II, Fig. 4), although it was not significantly different.

\section{Discussion}

We have previously reported that some antitumor drugs, such as taxanes and cyclophosphamide, and X-ray irradiation upregulated dThdPase in tumor tissues. We have also reported that capecitabine in combination with these dThdPase upregulators showed a synergistic antitumor activity in human cancer xenograft models (6-8). In the present study, the combination of capecitabine and oxaliplatin showed supraadditive antitumor activity in human gastrointestinal cancer xenograft models. The combination therapy of capecitabine at 2/3 MTD with oxaliplatin at 2/3 MTD showed significantly higher antitumor activity than each monotherapy at MTD in

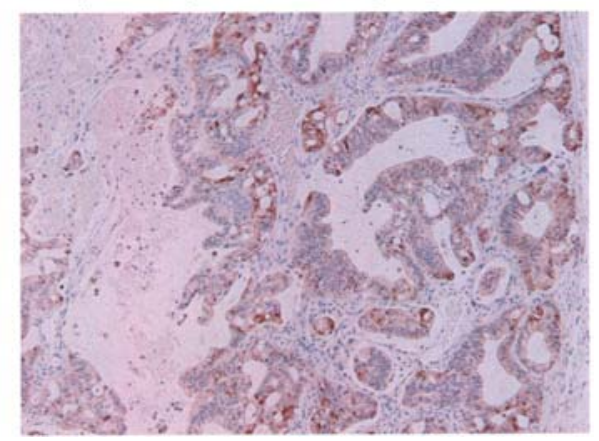

Figure 4. Immunohistochemistry for dThdPase in tumor tissues treated with vehicle (4a) and oxaliplatin $10 \mathrm{mg} / \mathrm{kg}$ (4b) on Day 4. Oxaliplatin (10 mg/kg) was administered intravenously into human colon cancer xenograft COL-05-JCK. 
Table II. Up-regulation of dThdPase by oxaliplatin.

\begin{tabular}{lcc}
\hline Sample & $\begin{array}{c}\text { dThdPase level }{ }^{\mathrm{a}} \\
\text { (unit/mg protein) (fold) }\end{array}$ & $\begin{array}{c}\text { Ratio of dThdPase- } \\
\text { positive cells }{ }^{\mathrm{b}} \\
(\%)(\text { fold })\end{array}$ \\
\hline $\begin{array}{l}\text { Day 4 } \\
\text { Vehicle }\end{array}$ & $9.7 \pm 1.5(-)$ & $2.9 \pm 3.9(-)$ \\
Oxaliplatin & $11.5 \pm 3.0(1.2)$ & $11.7 \pm 5.5(4.0)$ \\
$10 \mathrm{mg} / \mathrm{kg}$ & & \\
& & \\
Day 7 & & $4.1 \pm 1.7(-)$ \\
Vehicle & $8.2 \pm 0.6(-)$ & $11.2 \pm 9.4(2.7)$ \\
Oxaliplatin & $11.5 \pm 4.1(1.4)$ & \\
$10 \mathrm{mg} / \mathrm{kg}$ & & \\
\hline
\end{tabular}

Oxaliplatin was administered intravenously into human colon cancer xenograft model COL-05-JCK. Tumor tissues were excised to measure levels of dThdPase-IHC at 4 days and 7 days after

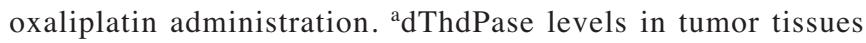
measured by ELISA. ${ }^{b}$ The area of dThdPase-positive cells stained in IHC as described in Materials and methods.

the human colorectal cancer CXF280 xenograft model. Additionally, no augmentation of toxicity regarding body weight loss was observed in the combination.

Furthermore, oxaliplatin up-regulated the level of dThdPase in tumor tissue without increasing the level of dThdPase in the liver (data not shown). dThdPase is a key enzyme necessary for capecitabine to exert its antitumor activity, and its level in tumor tissue has been reported to correlate with the antitumor activity of capecitabine, not of 5-FUra. Therefore, the tumor-specific up-regulation of dThdPase by oxaliplatin is considered to be one of the mechanisms for supra-additive antitumor activity, without the augmentation of toxicity observed in the combination of capecitabine and oxaliplatin. This synergy might not occur with the combination of oxaliplatin and 5-FUra/LV.

The mechanism of the tumor-specific up-regulation of dThdPase by oxaliplatin and other treatments has not been clarified. It has been reported that dThdPase confers resistance to apoptosis (14), and that tumor cells would acquire survival advantages by up-regulating dThdPase to counteract apoptosis induced by antitumor treatments. Further investigation of the mechanism of tumor-specific up-regulation of dThdPase by antitumor treatments is warranted in the future.

In the COL-05-JCK model, the efficacy of the combination was significantly superior to that of each monotherapy in terms of the delay of tumor growth. Expression of dThdPase in tumor cells after treatment with oxaliplatin tended to be up-regulated according to the IHC of this model, although the up-regulation determined by ELISA was not significant. These results suggest that oxaliplatin would potentiate the antitumor activity of capecitabine in this model as well. To investigate the possibility that capecitabine might enhance the efficacy of oxaliplatin, we examined another administration schedule of capecitabine treatment for 14 days, followed by oxaliplatin on day 7 or 15 of the treatment. The antitumor activity of oxaliplatin in this schedule was not augmented by the pretreatment of capecitabine (data not shown).

In the present pre-clinical study, the combination therapy of capecitabine with oxaliplatin showed superior antitumor activity at the lower dose of 2/3 MTD. It is suggested that the up-regulation of dThdPase by oxaliplatin in tumor tissue would allow a dose reduction of capecitabine with potentiation of antitumor activity. A recent Phase II study of the combination therapy of capecitabine and oxaliplatin for metastatic colorectal cancer showed a favorable safety profile of response rates, time to progression, and overall survival (11). Further clinical study using the combination of capecitabine and oxaliplatin is warranted for colorectal and gastric cancers.

\section{References}

1. Miwa M, Ura M, Nishida M, et al: Design of a novel oral fluoropyrimidine carbamate, capecitabine, which generates 5fluorouracil selectively in tumours by enzymes concentrated in human liver and cancer tissue. Eur J Cancer 34: 1274-1281, 1998.

2. Hoff PM, Ansari R, Batist G, et al: Comparison of oral capecitabine versus intravenous fluorouracil plus leucovorin as first-line treatment in 605 patients with metastatic colorectal cancer: results of a randomized phase III study. J Clin Oncol 19: 2282-2292, 2001

3. Van Cutsem E, Twelves C, Cassidy J, et al: Oral capecitabine compared with intravenous fluorouracil plus leucovorin in patients with metastatic colorectal cancer: results of a large phase III study. J Clin Oncol 19: 4097-4106, 2001.

4. Scheithauer W, McKendrick J, Begbie S, et al: Oral capecitabine as an alternative to i.v. 5-fluorouracil-based adjuvant therapy for colon cancer: safety results of a randomized, phase III trial. Ann Oncol 14: 1735-1743, 2003.

5. Ishikawa T, Sekiguchi F, Fukase Y, et al: Positive correlation between the efficacy of capecitabine and doxifluridine and the ratio of thymidine phosphorylase to dihydropyrimidine dehydrogenase activities in tumors in human cancer xenografts. Cancer Res 58: 685-690, 1998.

6. Sawada N, Ishikawa T, Fukase Y, et al: Induction of thymidine phosphorylase activity and enhancement of capecitabine efficacy by taxol/taxotere in human cancer xenografts. Clin Cancer Res 4: 1013-1019, 1998.

7. Endo M, Shinbori N, Fukase Y, et al: Induction of thymidine phosphorylase expression and enhancement of efficacy of capecitabine or 5'-deoxy-5-fluorouridine by cyclophosphamide in mammary tumor models. Int J Cancer 83: 127-134, 1999.

8. Sawada N, Ishikawa T, Sekiguchi F, et al: X-ray irradiation induces thymidine phosphorylase and enhances the efficacy of capecitabine (Xeloda) in human cancer xenografts. Clin Cancer Res 5: 2948-2953, 1999.

9. De Gramont A, Figer A, Seymour M, et al: Leucovorin and fluorouracil with or without oxaliplatin as first-line treatment in advanced colorectal cancer. J Clin Oncol 18: 2938-2947, 2000.

10. Rothenberg ML, Oza AM, Bigelow RH, et al: Superiority of oxaliplatin and fluorouracil-leucovorin compared with either therapy alone in patients with progressive colorectal cancer after irinotecan and fluorouracil-leucovorin: interim results of a phase III trial. J Clin Oncol 21: 2059-2069, 2003.

11. Cassidy J, Tabernero J, Twelves C, et al: XELOX (capecitabine plus oxaliplatin): active first-line therapy for patients with metastatic colorectal cancer. J Clin Oncol 22: 2084-2091, 2004.

12. Nishida M, Hino A, Mori K, et al: Preparation of anti-human thymidine phosphorylase monoclonal antibodies useful for detecting the enzyme levels in tumor tissues. Biol Pharm Bull 19: 1407-1411, 1996.

13. Sawada N, Nose T, Ishikawa T, et al: Enhancement of antitumor activity of 5'-deoxy-5-fluorouridine (Furtulon) by taxane in human gastric cancer xenografts. Oncol Rep 14: 53-57, 2005.

14. Ikeda R, Furukawa T, Mitsuo R, et al: Thymidine phosphorylase inhibits apoptosis induced by cisplatin. Biochem Biophys Res Commun 301: 358-363, 2003. 